\title{
Role of Radiology in Tropical Diseases
}

\author{
Dr. Maulik Jethva ${ }^{1}$, Dr. Manisha Panchal ${ }^{2}$ \\ ${ }^{1}$ (Radiology Department, PDU Govt. Medical College, Rajkot, Gujarat, India) \\ ${ }^{2}$ (Medicine Department, PDU Govt. Medical College, Rajkot, Gujarat, India)
}

\begin{abstract}
Tropical diseases are infectious diseases that are prevalent in or unique to tropical and subtropical regions. India is a tropical country \& these diseases permanently curtail human potential, anchoring at least one billion poor people in poverty. They cause enormous economic burden for endemic countries. All such diseases cause great human misery. So this research work is done to evaluate useful role of radiology in early diagnosis of such tropical diseases \& to assess value of various radiological modalities like X-rays, Ultrasonography, CT Scan, MR imaging etc. in diagnosis of such tropical diseases with a cost effective approach.
\end{abstract}

Keywords: Tropical Radiology, Tropical Diseases, Radiology in Tropical diseases.

\section{INTRODUCTION}

Tropical diseases are infectious diseases that are prevalent in or unique to tropical and subtropical regions. ${ }^{[1]}$ India is a tropical country and these diseases cause great human misery with enormous economic burden for endemic countries. Human exploration of rainforests and increased international air travel and other tourism to tropical regions has led to an increased incidence of such diseases. ${ }^{[2]}$ The greenhouse effect and increasing global temperature of the atmosphere seem to be influencing the spread of tropical diseases and vectors to other latitudes that were previously spared them, such as Southern United States, the Mediterranean areas etc. ${ }^{[1]}$ An estimated 1 billion people - one sixth of the world populations - are infected with one or more of these diseases, ${ }^{[2]}$ so it is important to early diagnose these diseases. Various radio-diagnostic modalities like Xrays, Ultrasonography, CT scan, MR imaging etc. can early diagnose most of these diseases, so it is important to know that which modality/modalities is/are useful for us for a definite diagnosis with a cost effective approach.

\section{AIMS AND OBJECTIVES}

- To evaluate the role of radiology in diagnosis of Tropical diseases.

- To assess the value of correlative radiological imaging with a cost effective approach.

\section{MATERIALS AND METHODS}

In this study, we have collected cases \& data of 50 cases of tropical diseases during the period of June 2006 to October 2008, during the period of our postgraduate residency. Most of the patients were referred from medical \& surgical departments of civil hospital - Ahmadabad. The patients were selected on the basis of either clinical provisional diagnosis of tropical disease or laboratory-pathology investigations were indicative in favor of the tropical disease. All the patients were examined by one or more radio-diagnostic imaging modality as required; required X-rays \& USG of almost all patients were done. Additional radiological investigations like contrast X-ray procedure, CT scan, MRI etc. whichever needed was done to approach the definite diagnosis. Follow up of all patients were also done during and after treatment.

\section{Classifications Of Tropical Diseases}

There are many ways to classify the Tropical diseases like: Disease wise classification, System wise (Body system affected) classification and the WHO classification. Among all these, "Disease wise classification" is more commonly used, so here mentioned. ${ }^{[3]}$

1) Parasitic diseases: (1a) Major multisystem diseases - Amebiasis, Hydatid disease, Schistosomiasis, Taeniasis, Chagas disease \& Paragonimasis. (1b) Gastrointestinal and abdominal diseases - Predominantly small bowel involving - Ascariasis, Strongyloidiasis, Ancylostomiasis, Anisakiasis, Capillariasis \& Giardiasis. Predominantly colon involving - Trichriasis \& Helminthoma. Liver involving - Clonorchiasis, liver flukes \& Fascioliasis. (1c) Soft tissue diseases - Drancuncliasis \& Filarial diseases. (1d) Miscellaneous diseases - Malaria, Dengue, Tropical splenomegaly \& Kala azar.

2) Non-Parasitic diseases: (2a) Major multisystem diseases - AIDS, Tuberculosis \& Mycoses. (2b) Gastrointestinal and abdominal diseases - Predominantly small bowel involving - Typhoid \& Paratyphoid fevers. Predominantly colon involving - Bacillary Desentery \& Lymphogranuloma Venereum. (2c) Soft tissue \& Bone diseases - Soft tissue involving - Tropical Pyomyositis. Bone involving - Cancrum Oris, 
Brucellosis, Tropical ulcer, Leprosy, Tetanus, Small Pox, Scleroma. Chest/Lung involving - Plague \& Melioidosis.

3) Neoplastic (Malignant) diseases: Burkitt's lymphoma \& Kaposi Sarcoma.

4) Miscellaneous diseases: Gastrointestinal - Tropical Sprue, CVS involving - Anaemias, Chagas disease, Endomyocardial fibrosis, CHD, Atheroma, IHD \& Pulmonary hypertension etc. Soft tissue involving Tumoral calcinosis. Bone involving - Ainhum, Haemoglobinopathies etc.

- The WHO classification ${ }^{[1,4]}$ includes following common entries: African Trypanosomiasis, Dengue fever, Leishmaniasis, Malaria, Schistosomiasis, Tuberculosis, Chagas disease, Leprosy, Lymphatic Filariasis \& Onchocerciasis. Additional neglected tropical diseases includes ${ }^{[1,2]}$ : Ascariasis, Trichuriasis, Hookworm, Trachoma, Dracunculiasis, Buruli ulcer, Treponematoses, Leptospirosis, Strongyloidiasis, Neurocysticercosis, Scabies. Some very rare diseases may occur in sudden epidemics are: Ebola hemorrhagic fever, Lassa fever \& the Marburg viral disease.

\section{OBSERVATIONS AND ANALYSIS}

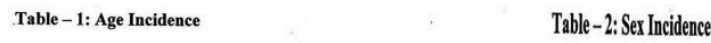

\begin{tabular}{|c|c|c|}
\hline Age (Yrs) & No. of Patient & Percentage \\
\hline$\triangleleft /=10$ & 6 & 12 \\
\hline $11-20$ & 5 & 10 \\
\hline $21-30$ & 12 & 24 \\
\hline $31-40$ & 10 & 20 \\
\hline $41-50$ & 8 & 16 \\
\hline $51-60$ & 7 & 14 \\
\hline $61-70$ & 2 & 4 \\
\hline & 50 & $100 \%$ \\
\hline
\end{tabular}

\begin{tabular}{|c|c|c|}
\hline Sex & No. of Patient & Percentage \\
\hline Male & 30 & 60 \\
\hline Female & 20 & 40 \\
\hline & 50 & $100 \%$ \\
\hline
\end{tabular}

Table - 1: Shows Age incidence of the patients which were affected by Tropics diseases. It shows that affected maximum patients (24\%) were between $21-30 \mathrm{yrs}$.

Table - 2: Shows those predominantly male patients were affected more than female

patients by tropical diseases. Male: Female ratio is 3:2.

Table -3: Parasitic VS Non Parasitic Diseases

\begin{tabular}{|c|c|c|}
\hline & No. of Patient & Percentage \\
\hline Non Parasitic Diseases & 28 & 56 \\
\hline Parasitic Diseases & 22 & 44 \\
\hline & 50 & $100 \%$ \\
\hline
\end{tabular}

Table - 3: Shows that incidence of Non- Parasitic diseases (56\%) were more than Parasitic diseases (44\%) in the cases.

Table - 4: Disease Incidence

\begin{tabular}{|c|c|c|}
\hline Disease & No. of Patient & Percentage \\
\hline Tuberculosis & 13 & 26 \\
\hline HIV Infection & 9 & 18 \\
\hline Hydatid Disease & 7 & 14 \\
\hline Worm Infections & 5 & 10 \\
\hline Cysticerosis & 3 & 6 \\
\hline Amocbiasis & 3 & 6 \\
\hline Haemoglobinopathies & 3 & 6 \\
\hline Tropical Spleenomegaly & 2 & 4 \\
\hline Leprosy & 2 & 4 \\
\hline Typhoid & 1 & 2 \\
\hline Schistosomiasis & 1 & 2 \\
\hline Toxoplasmosis & 1 & 2 \\
\hline & 50 & $100 \%$ \\
\hline
\end{tabular}

Table - 4: Shows that Tuberculosis (26\%) and HIV infection (18\%) were affected majo proportion of overall cases.

\section{Table - 5: Incidence of Multi system Diseases}

\begin{tabular}{|c|c|c|}
\hline Disease & No. of Patient & Percentage \\
\hline Tuberculosis & 13 & 26 \\
\hline HIV Infection & 9 & 18 \\
\hline Hydatid Disease & 7 & 14 \\
\hline Taeniasis, Cysticercosis & 4 & 8 \\
\hline Amoebiasis & 3 & 6 \\
\hline Schistosomiasis & 1 & 2 \\
\hline Total & 37 & $74 \%$ \\
\hline
\end{tabular}

Table - 5: According to classification, in the category of Major multi-system diseases, Tuberculosis (26\%) and HIV infection (18\%) were affected major proportion of overall cases. Total overall incidence of Multi-system diseases was $74 \%$.
Table - 6: Incidence of Organ/System Involvement (Multiple OrganSystem involvement by a single discese is also taken into consideration.

\begin{tabular}{|c|c|c|}
\hline Organ/System & No. of Cases & Percentage \\
\hline Lungs & 13 & 26 \\
\hline Liver & 11 & 22 \\
\hline Bowel (GII) & 11 & 22 \\
\hline Skeletal System & 11 & 22 \\
\hline Brain (CNS) & 8 & 16 \\
\hline Spleen & 6 & 12 \\
\hline Heart (CVS) & 4 & 8 \\
\hline Kidneys, Bladder (GUT) & 3 & 6 \\
\hline Penitoneum & 3 & 6 \\
\hline Lymphnodes & 3 & 6 \\
\hline Odbit & 1 & 2 \\
\hline
\end{tabular}

Table -6: Incidence of Organ/System involvement shows that Lungs (Respi.) were most commonly (26\%) affected and Liver, Bowel (GIT) and Skeletal system were second (22\%) commonly affected by Tropical diseases. 


\begin{tabular}{|c|c|c|}
\hline \multicolumn{3}{|c|}{$\begin{array}{l}\text { Table-7: Incidence of Positive findings in } \\
\text { Various Radidological modallities }\end{array}$} \\
\hline Radilogigel modality & No.oflesess & Percentage \\
\hline$X$-Rass & 30 & 60 \\
\hline Utrasanography & 26 & 52 \\
\hline CTSan & 21 & 42 \\
\hline MRI & 8 & 16 \\
\hline Coverational Contras Procedurs & 1 & 14 \\
\hline Other Radiongial Modilities & . & . \\
\hline
\end{tabular}

Table-7: Plain X-Rays wee positive in 60\% of case and USO wasp positure in $52 \%$ of coses. Both the invetigations have very high positive predictive values

Table - 10: Diagnosed Cases mainly by: Conventional Contrast Procedures

\begin{tabular}{|c|c|c|c|}
\hline $\begin{array}{c}\mathrm{Sr} \\
\mathrm{No} \text {. }\end{array}$ & Case No. & Disease & OrganSSystem involved \\
\hline 1 & 9 & Tuberculosis & GII \\
\hline 2 & 10 & Tuberculosis & GI, Respi. \\
\hline 3 & 15 & Round Worm & GII \\
\hline 4 & 16 & Tape Wom & GII \\
\hline 5 & 17 & Tuberrulosis & GI \\
\hline 6 & 37 & Amebic Colitis & GII, Liver \\
\hline
\end{tabular}

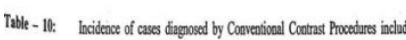

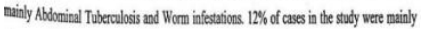

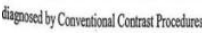

Table - 8: Incidence of the Last Radiological modality Which reaches up to the Final Diagnosis

\begin{tabular}{|c|c|c|}
\hline Radiological modality & No. of Cases & Percentage \\
\hline CT Scan & 19 & 38 \\
\hline X-Rays & 10 & 20 \\
\hline MRI & 8 & 16 \\
\hline Ultrasonography & 7 & 14 \\
\hline Conventional Contrast Procedures & 6 & 12 \\
\hline Other Radiological Modalities & $\cdot$ & $\cdot$ \\
\hline & 50 & $100 \%$ \\
\hline
\end{tabular}

Table -8: Shows that CT Scan was used mainly (38\%) as the last radiological modality to reach the final diagnosis.

Table - 11: Diagnosed Cases mainly by: Ultrasonography

\begin{tabular}{|c|c|c|c|}
\hline Sr. & Case No. & Disease & Organ/System involved \\
No. & & & \\
\hline 1 & 13 & Amebiasis & GI, Liver \\
\hline 2 & 28 & HIV Infection & Spleen \\
\hline 3 & 29 & Malaria & Spleen \\
\hline 4 & 30 & Amebiasis & Liver \\
\hline 5 & 32 & Typhoid & GIT \\
\hline 6 & 38 & Malaria & Spleen \\
\hline 7 & 39 & Round Worm & GIT \\
\hline
\end{tabular}

Table - 11: Incidence of cases diagnosed by Ultrasonography includes mainly Amebic Table - 12: Incidence of cases mainly diagnosed by MRI includes Tuberculosis, HIV Liver abseess and Tropical splennomegaly in Malaria 14\% of cases in the study were Encephalitis, Cysticercosis and Toxoplasmosis. $16 \%$ of cases in the study were mainly mainly diagnosed by Ultrasonography.
Table - 9. Diagnosed Cases mainly by: Plain X-Rays

\begin{tabular}{|c|c|c|c|}
\hline $\begin{array}{c}\text { Sr. } \\
\text { No. }\end{array}$ & Case No. & Disease & Organ/System involved \\
\hline 1 & 8 & Tuberculosis & Skeletal Sys, Lymphnodes \\
\hline 2 & 11 & Guinea worms & Skeletal Sys. \\
\hline 3 & 12 & Guinea worms & Skeletal Sys. \\
\hline 4 & 33 & Leprosy & Skeletal Sys. \\
\hline 5 & 40 & PCP (HIV) & Respi. Sys. \\
\hline 6 & 42 & Sickle cell disease & Skeletal Sys, CVS \\
\hline 7 & 43 & Thalassemia & Skeletal Sys, CVS, Liver, Spleen, Kidney \\
\hline 8 & 44 & Thalassemia & Skeletal Sys, CVS, Liver, Spleen \\
\hline 9 & 45 & Schistosomiasis & Kidneys, Bladder (GUT) \\
\hline 10 & 50 & Leprosy & Skeletal Sys. \\
\hline
\end{tabular}

Table - 9: Incidence of Plain X-Ray diagnosed cases includes mainly Haemoglobinopathis, Leprosy and Guinea Worm infection. $20 \%$ of cases in the study were mainly Diagnosed by Plain X-Rays

Table - 12: Diagnosed Cases mainly by: MR

\begin{tabular}{|c|c|c|c|}
\hline $\begin{array}{r}\text { Sr. } \\
\text { No. }\end{array}$ & Case No. & Disease & Organ/System involved \\
\hline 1 & 2 & Tuberculosis & CNS, Respi. \\
\hline 2 & 14 & Cysticercosis & Skeletal Sys. \\
\hline 3 & 18 & Tuberculosis & CNS, Respi. \\
\hline 4 & 21 & Toxoplasmosis & CNS, Respi. \\
\hline 5 & 31 & Tuberculosis & Skeletal Sys. \\
\hline 6 & 34 & Cysticercosis & CNS \\
\hline 7 & 35 & HIV Encephalitis & CNS, Respi. \\
\hline 8 & 46 & HIV Encephalopathy & CNS \\
\hline
\end{tabular}
diagnosed by MRI.

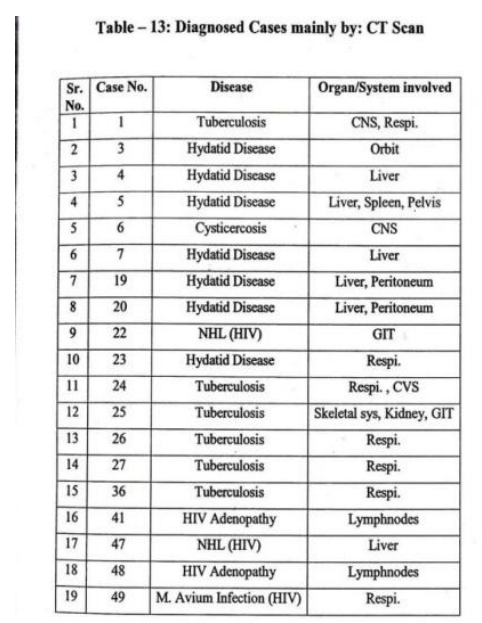

Table - 13: Incidence of cases diagnosed by CT Scan includes mainly Hydatid disease and Tuberculosis. Highest numbers of cases (38\%) in the study were mainly diagnosed by CT scan.

\section{SUMMARY AND CONCLUSION}

- Maximum patients (24\%) were affected by Tropical diseases between ages $21-30$ yrs. Tropical disease which affected mainly above mentioned age group were: Tuberculosis, HIV infection \& Hydatid disease.

- Predominantly male patients $(60 \%)$ were affected more than female patients $(40 \%)$ by Tropical diseases. Male: Female ratio was 3:2.

- Non-Parasitic diseases (56\%) had more incidence than Parasitic diseases (44\%) in the cases because of high incidences $(44 \%)$ of Tuberculosis \& HIV infections.

- Tuberculosis $(26 \%)$ \& HIV infection (18\%) were affected major proportion (44\%) of overall cases.

- Worm infections including Cysticercosis $(16 \%)$ \& Hydatid disease $(14 \%)$ were more common in the category of Parasitic diseases in the cases.

- Lungs (Respiratory system) were most commonly (26\%) affected by Tropical diseases.

- Plain X-rays were positive in $60 \%$ of cases and USG was positive in $52 \%$ of cases. Both the investigations are currently used as most common primary radiological imaging modalities and have high positive 
predictive values. So these modalities either suggest approximate diagnosis or gives clues for further investigations.

- USG guided diagnostic/therapeutic aspiration procedures have major advantage in the treatment as real time, radiation less \& cost effective imaging approach.

- Highest numbers of cases (38\%) in the study were mainly diagnosed by CT scan as the last radiological modality to reach the final diagnosis.

- As MRI study is the preferred modality for detail evaluation and differential diagnosis, major limiting factors are their cost and limited availability in poorly developed tropical areas. In this study, combined diagnostic accuracy of X-rays, USG \& CT scan were $84 \%$. So, this suggests that combined use of other available cost effective radiological modalities like X-rays, USG \& CT scan reduces the need of costly MRI study.

Thus, as per the objectives, the present study has evaluated the role of radiology in investigations and diagnosis of Tropical diseases and justified the values of correlative imaging with a cost effective approach.

\section{REFERENCES}

[1]. Tropical diseases: From Wikipedia, the free encyclopedia

[2]. Hotez, P. J.; Molyneux, DH; Fenwick, A; Kumaresan, J; Sachs, SE; Sachs, JD; Savioli, L (September 2007). "Control of Neglected Tropical Diseases". The New England Journal of Medicine 357 (10): 1018-1027. doi:10.1056/NEJMra064142. ISSN 00284793. PMID 17804846. 17804846. Retrieved 2008-01-21.

[3]. Medcyclopaedia: Tropical Diseases, Chapter: 27, By Philip E.S. Palmer with Stanley P. Bohrer, Carlos Bruguera, Xing-Rong Chen, Mahmoud R. Elmeligi, Hassen A. Gharbi, S.B. Lagundoye, M.W. Wachira.

[4]. "Disease portfolio". Special Program for Research and Training in Tropical Diseases: The World Bank and the World Health Organization. Retrieved 2009-08-01. 